\title{
Multi-length Windowed Feature Selection for Surface EMG based Hand Motion Recognition
}

\author{
Dalin Zhou, Yinfeng Fang, Zhaojie Ju, and Honghai Liu \\ Intelligent Systems \& Biomedical Robotics Group, School of Computing, \\ University of Portsmouth, Portsmouth PO1 3HE, UK \\ \{dalin.zhou, yinfeng.fang, zhaojie.ju, honghai.liu\}@port.ac.uk
}

\begin{abstract}
Feature selection for surface electromyography based hand motion recognition has been seen to retrieve an optimal or quasi-optimal feature subset for classification. This work aims to consider the influence of channel, feature and window length simultaneously with an emphasis on the multiple segmentation. The bacterial memetic algorithm is applied to select the feature candidates from time domain and autoregressive coefficients, which is measured by the inter-day hand motion recognition accuracy. The evaluation is conducted on a case study of 3 able-bodied subjects performing 9 hand motions in consecutive 7 days with 4 different window lengths adopted for the electromyographic data segmentation. Classification in combination with the multi-length windowed feature selection achieved an improved recognition accuracy in comparison with using solely the single-length windowed features in inter-day scenarios and indicated that complementary information to full length segmentation resides in the sub-windows, thus providing feasible feature combinations for conventional pattern recognition based solutions to prosthetic control.
\end{abstract}

Keywords: Electromyography, Hand Motion Recognition, Window Segmentation, Feature Selection, Bacterial Memetic Algorithm

\section{Introduction}

Hand motions play a vital role in activity of daily livings and the recognition of hand motion is always a trending research topic. Besides the contribution towards a better recognition of hand motions for able-bodied people in the humanhuman and human-object interaction [1], active demands exist for subjects with limb impairment who would benefit more from improved hand motion recognition and execution. As a result, recent decades have witnessed the prompt development of the prosthetic hand design and its corresponding control strategies [2]. Among all the feasible noninvasive sensing modalities, surface electromyography (sEMG) has remained the mainly adopted access to upper limb motion intention for its simplicity and robustness [3]. An accurate recognition of motion intention forms the basis of an intuitive control of the prosthesis which has been intensively investigated in this field. The most prominent recognition results in prosthetic 
control with a promising recognition accuracy rate over $90 \%$ are mostly confined to pattern recognition based solutions in laboratory environment. Despite the most recently growing research interest in deep learning approaches $[4,5]$, conventional pattern recognition based methods with handcrafted features still play a dominant role in both academic and clinical scenarios. As a result, different stages of a typical pattern recognition problem have been continuously studied in the case of sEMG based hand motion recognition including signal preprocessing, feature extraction, classification and postprocessing. The success of a pattern recognition application empirically relies on the premise that distinguishable and consistent features are extracted. The importance of an effective feature set in sEMG based motion recognition has also been confirmed by Scheme et al. [6] with the observation of similar performance over most modern classifiers. Thus the sEMG feature extraction in domains of time, frequency and time-frequency has been elaborated on, as per the evaluation conducted by Phinyomark et al. [7].

Besides the numerous research of feature extraction strategies, feature selection is also addressed by researchers seeking for an optimised feature combination. Oskoei et al. [8] applied the genetic algorithm in the recognition of 6 motions to select the optimal subset for their 4-channel myoelectric system, while Khushaba et al. utilised the particle swarm optimisation to reduce the dimension of feature and channel combinations [9]. Al-Timemy et al. [10] looked into the finger movement classification and achieved comparable accuracy with subsets of selected channels instead of utilising the full set of all channels. Recent research has been seen on the feature selection against force and position variation. The classification accuracy under limb variation was improved by [11] with the selected subset out of 10 feature candidates. Adewuyi et al. [12] evaluated the optimal sEMG feature subset in varying wrist positions for subjects with partial hand amputation and proved its superiority over the ensemble TD or TDAR features. Let alone the emphasis on the feature selection, the importance of the data segmentation with various window length of sEMG stream is addressed by [13]. However to our knowledge, no studies have yet investigated the feature subset selection with fused multiple window lengths. The subset selection that simultaneously concerns feature type, detecting site and window segmentation is still missing. In our previous work, a memetic evolutionary method named bacterial memetic algorithm (BMA) has been applied in reducing the feature dimension while preserving a comparable recognition accuracy [14]. To further explore an optimised feature subset considering the three factors mentioned above, a BMA based feature selection of feature candidates extracted from multi-length windows is evaluated in this paper.

The rest of this paper is organised as follows. A brief introduction of the conventional routine of sEMG based hand motion recognition and the BMA based feature selection is outlined in Section 2. And the experiment setup and results are presented and further discussed in Section 3. After the discussion, this paper is finally concluded in Section 4 . 


\section{EMG Feature Extraction and Selection}

\subsection{Handcrafted Feature Extraction}

A typical conventional pattern recognition flowchart for sEMG based hand motion recognition is illustrated in Fig. 1. Raw sEMG signals are first captured by either dry or wet electrodes attached on the skin surface of upper limbs. Filters are then adopted to remove common mode noises, power line noises and irrelevant components before feeding the signal stream to subsequent pattern recognition system. The stream is later routinely segmented by windows with either overlapping or non-overlapping increments. For each segment, its features are extracted and classified into predefined motion types, which forms the sequence of recognition results. Postprocessing techniques such as majority vote and velocity ramp are then used to concatenate the decision stream for prosthetic control. The assumption of this paper is that the combination of multiple segmentation and feature extraction strategies can be optimised to improve the classification results.

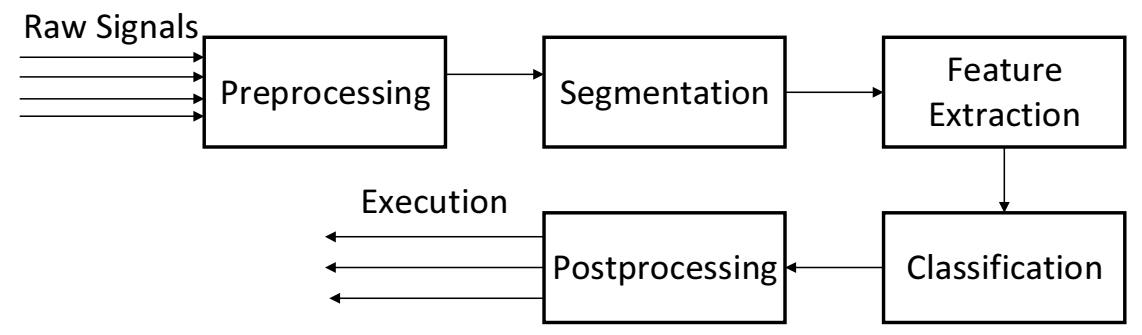

Fig. 1. A typical sEMG and pattern recognition based hand motion recognition

Despite the large amount of potential features, the Hudgins' time domain features [15] and the autoregressive coefficients (TDAR) have been mostly exploited for their less time consumption and robust performance, and remained the state-of-the-art for years. In this paper, mean absolute value (MAV), waveform length (WL), zero crossings (ZC), slope sign changes (SSC) and 4-th order autoregressive coefficients (AR) of multiple window lengths are incorporated as candidate features while segmentations of $250 \mathrm{~ms}, 200 \mathrm{~ms}, 150 \mathrm{~ms}$ and $100 \mathrm{~ms}$ are employed as the window lengths.

\subsection{Bacterial Memetic Algorithm}

BMA is an evolutionary memetic algorithm inspired by the nature of microbial evolution that combines both global and local optimisation [16]. Our previous work of feature selection for dimension reduction with BMA can be seen in [14] and a brief introduction of the memetic evolutionary technique is given 
here. BMA comprises basic operators including mutation, transfer and the local search, where they take advantages of both evolutionary and memetic approaches. The optimisation problem of feature selection is to seek the optimal or quasi-optimal combinations of features, which can be encoded by the chromosomes. And the evaluation of each chromosome is based on the predefined fitness which measures the accuracy for a classification problem. After the initialisation of random chromosomes, mutation, transfer and local search operators will function on the chromosomes according to the calculated fitness. The loop of three operations will be carried out continuously till the termination criteria are met. The chromosome with the best fitness will then be retrieved as the final output.

In this work, the chromosomes are encoded by nonnegative integers representing feature candidates and the pattern recognition based inter-day hand motion accuracy is adopted as the fitness. Similarly to our previous work, the chromosome length is constrained by the regulariser as follows [14] to avoid overfitting, where $\sigma_{i}$ is the fitness of $\mathrm{i}$-th chromosome/feature subset, $S$ is the number of hand motion samples for testing, $(x, t)$ is the sample from the testing domain $T, f(x)$ is the motion type classification which generates the labels, $L$ is the chromosome length, $U$ is the predefined upper boundary of chromosome length, $\lambda$ is the regularisation parameter and $\delta(k)$ is the Kronecker delta function.

$$
\begin{gathered}
\sigma_{i}=\frac{1}{S} \sum_{(x, t) \in T} \delta[f(x)-t]+\lambda \frac{L}{U} \\
\delta(k)= \begin{cases}1, & k=0 \\
0, & k \neq 0\end{cases}
\end{gathered}
$$

The mutation operator functions on every chromosome in each loop to import new information. Multiple duplications of the original chromosome will be created with subsequent mutation of random positions. The muted chromosomes together with the unaltered one will be evaluated. The original chromosome is then replaced by the one with the best fitness. In our application, the chromosome length is variable in terms of including new feature candidates or excluding the current ones, and the length variation will only take place in the mutation stage. The parameters required for this operation include the probabilities of length variation and mutation. The transfer operation exchanges the segments of two chromosomes to acquire more information from the superior one. Chromosomes will be first sorted and split into two halves according to their fitness, where the superior set comprises those with better fitness and vice versa. A pair of chromosomes are then randomly selected from the two sets and the pointwise exchange will be conducted on their fragments with a fixed length. The changes of chromosomes which lead to an inferior fitness will be eliminated. The two sets will be sorted again with their new fitness when an valid exchange occurs. The exchange position and length are the parameters required for this operation. The local search operator acts on each chromosome based on the prior knowledge in the application scenario. The local search regions are firstly defined according to the neighbour candidates of each feature. The evaluation is then conducted 
within the region to find the local optimum in each loop. In our research, the criteria of determining the searching region is confined within the detection site distribution and feature type. For example, the local search of a channel will be carried out among its proximate channels while the searching candidates of a feature type will remain in the same domain, which exploits the most of the clinical context and boosts the convergence rate.

\section{Experiment Setup and Analysis}

\subsection{Experiment Setup for Data Acquisition}

The sEMG capturing system developed by the authors [17] was used for the data acquisition. The system consists of 16 channels covering the forearm muscles on both anterior and posterior sides with the bi-polar electrodes embedded in a customised sleeve connected by wired cables as shown in Fig. 2. The acquisition system is equipped with a sampling frequency of $1000 \mathrm{~Hz}$, a gain of 3000 and an ADC resolution of 12 bits.
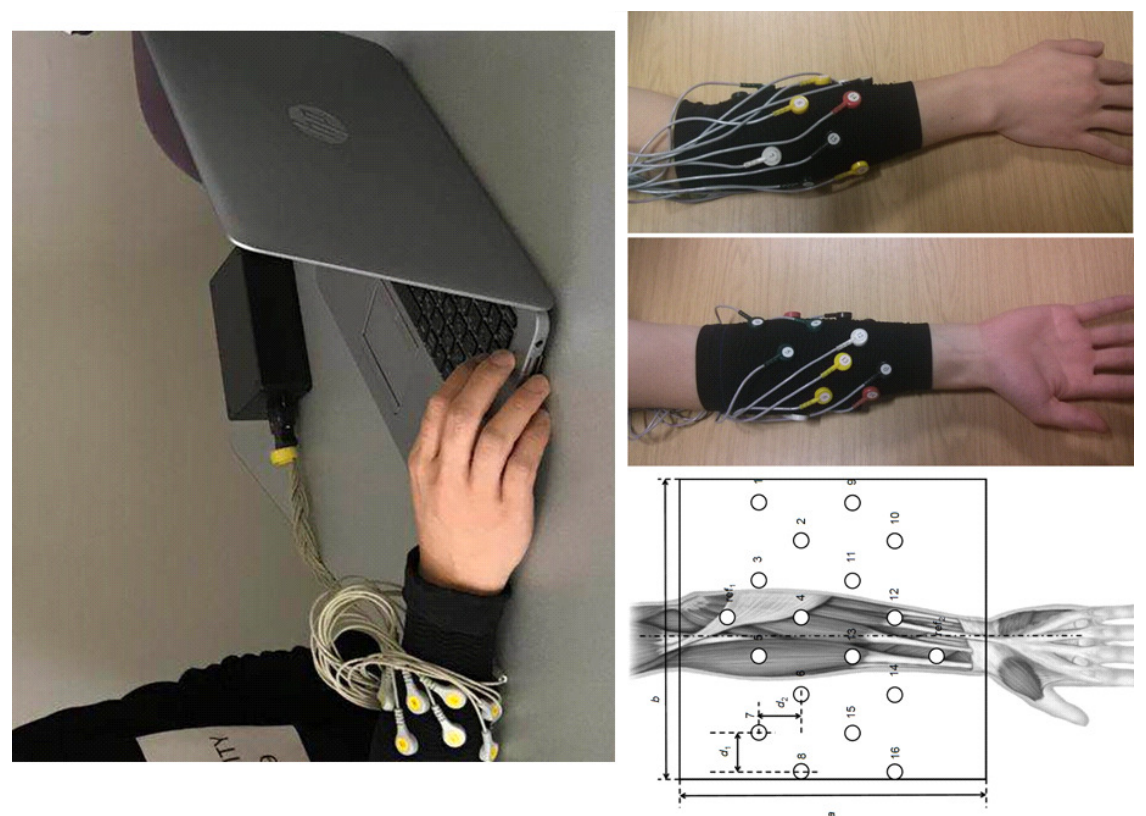

Fig. 2. A customised sEMG acquisition device [17]

Three able-bodied subjects participated in the experiment to conduct 9 hand motions including hand open/close, wrist extension/flexion, forearm supination/pronation, finger pinch/point and rest, as can be referred in Fig. 3. In 
this work, we evaluated the inter-day recognition and focused on the consistency of feature selection improvement. The training and validation data for feature selection came from the sEMG signals captured on one subject for 3 days while the test data was gathered from the other two subjects in consecutive 7 days to verify the feasibility. During the signal acquisition, subjects were asked to sit at their comfortable positions with elbows bent and forearm rest on the desk. Visual hints were displayed on the monitor to instruct the subjects of current motions to follow and each of them lasted for 10 seconds. A short rest was arranged between adjacent sessions to remedy the adverse effect of muscle fatigue.
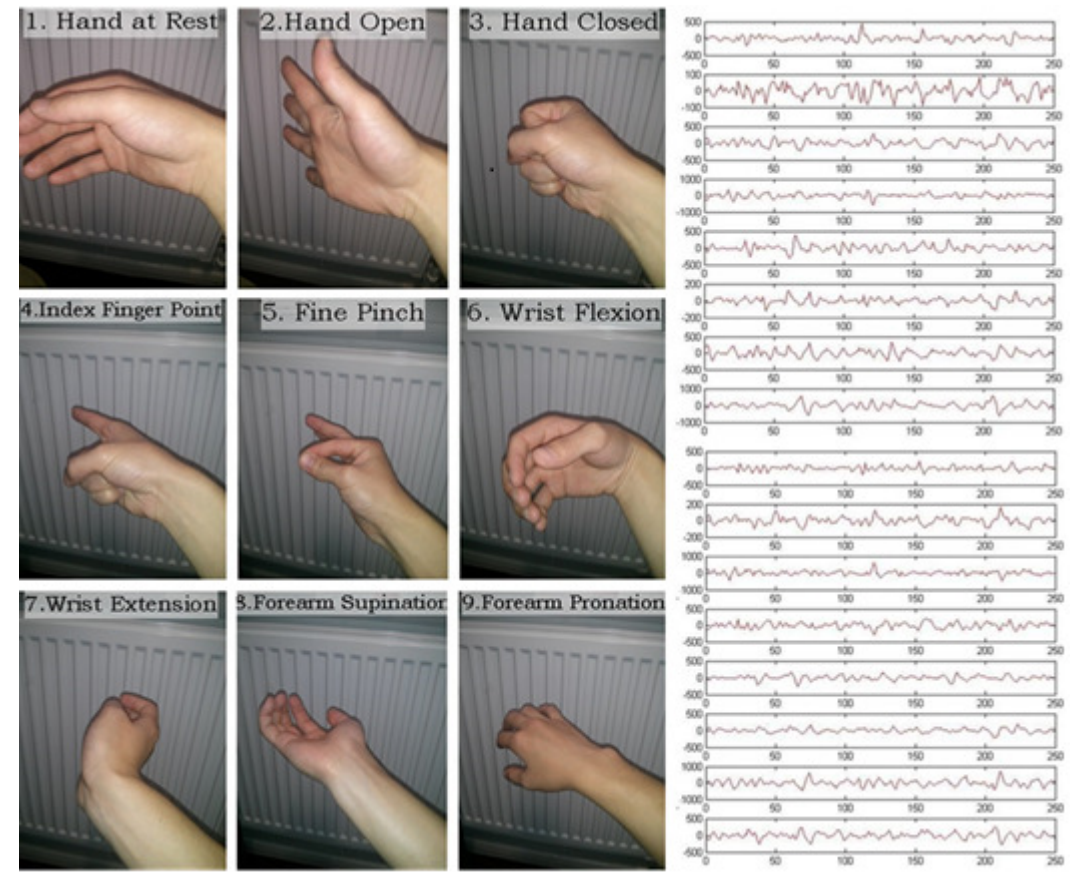

Fig. 3. Candidate motions and the 16-channel sEMG sample

The raw sEMG signals were firstly bandpass filtered by a Butterworth filter between $10 \mathrm{~Hz}$ and $500 \mathrm{~Hz}$ and a notch filter at the powerline frequency. Only the stationary phase of sEMG signals were kept for the recognition process the first and last 1 second of data between consecutive motions were discarded as the transient part. The filtered stationary stream was then segmented by the windows of $250 \mathrm{~ms}$ with an overlap of $200 \mathrm{~ms}$. The segmentations were further segmented with the length/overlap combinations of 200ms/190ms, 150ms/130ms and $100 \mathrm{~ms} / 50 \mathrm{~ms}$ as multi-length candidate sub-windows. The sub-windows were then concatenated and the feature extraction was performed on every one of them. For each window, a total of 128 dimensional features of 16 channels would 
be extracted which led to the final 2176 candidate features. Prior to the succeeding process, the features extracted on the same day were routinely normalised between 0 and 1 . To exploit the most statistically invariant information, the 128 features of the $250 \mathrm{~ms}$ window were kept as the basis while the selection was conducted among those sub-windows. The feature selection was carried out following the BMA with the inter-day recognition accuracy as fitness. Based on our prior knowledge in feature selection for sEMG based hand motion recognition, the parameter configuration of BMA followed the setting in [14] with a similar convergence shown in Fig. 4. Linear discriminant analysis (LDA) was adopted as the classifier and a preceding principal components analysis (PCA) was used to collect the first 20 components as the input. Despite the less strict time consumption requirement for offline applications like feature selection, the convergence of BMA is achieved within a small amount of iterations, which coincides with our previous research.

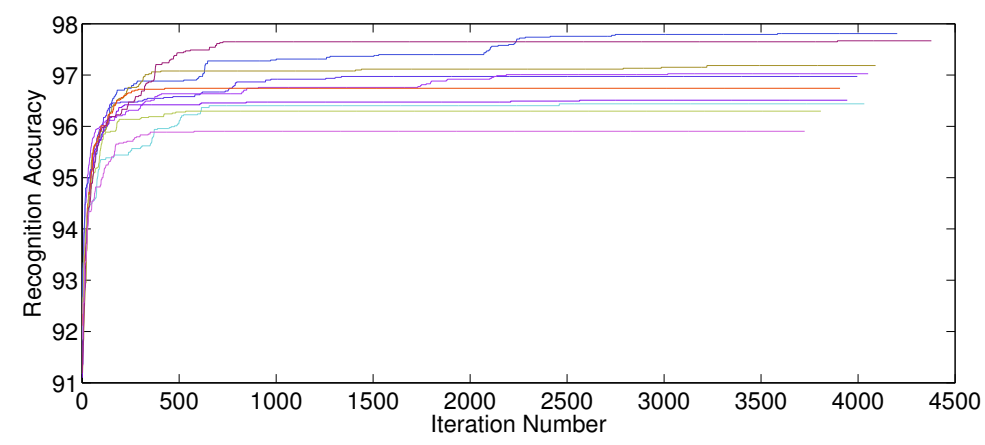

Fig. 4. Convergence for feature selection process

\subsection{Experiment Results and Discussion}

The feasibility of selected features is represented by inter-day recognition error respectively, on both testing and validating data from all three subjects in Fig. 5 , on the testing data only captured from the two subjects shown in terms of training on 1 day and testing on the rest 6 days in Fig. 6. The trend can be seen that the average errors decrease with the adoption of selected features in comparison with solely using the single-length windowed ones. However, a discrepant case occurs for subject 2 when the data on day 2 is adopted for training and tested on the remaining days.

The enumeration of the channels, window lengths and feature types selected are demonstrated in Fig. 7 respectively and summarised in Table 1. An intuitive selection preference can be deducted by the results that windows with a longer segment length are more likely to be selected. The TD features especially the 


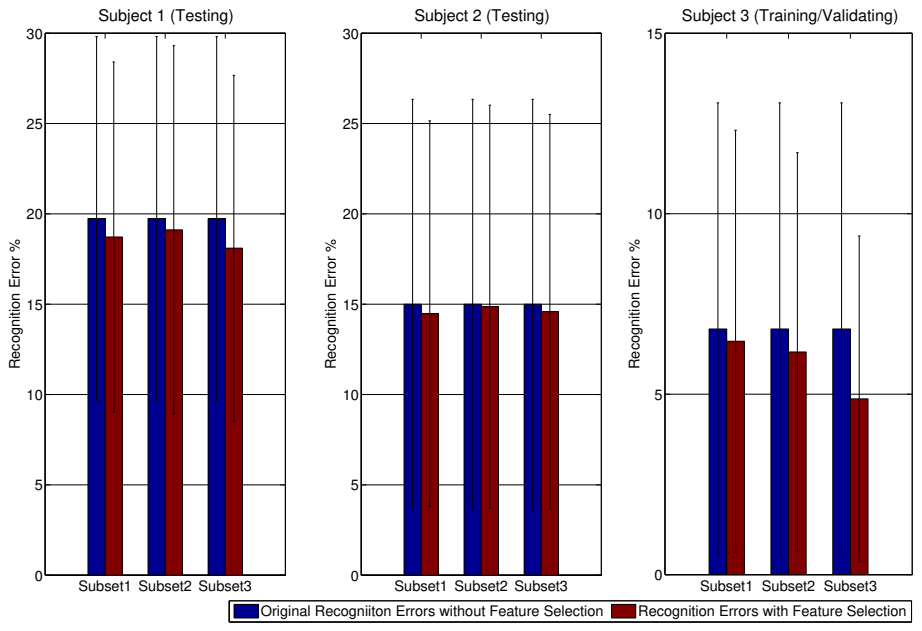

Fig. 5. Average recognition results with/without feature of 3 different subsets
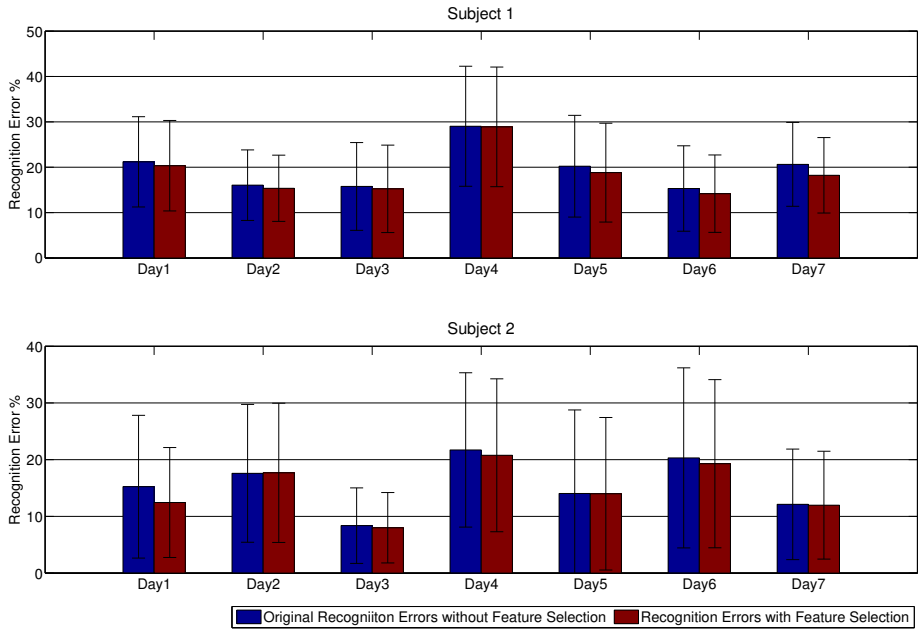

Fig. 6. Recognition with/without feature selection for inter-day use 
WL and SSC features and the first 2 components of the AR features are more favoured in the selection. The last $2 \mathrm{AR}$ components are least preferred during the selection. The preference towards a larger segmentation is seen when using accuracy as the evaluation criteria, which coincides with the previous research [18] that an increased window length will provide features with less statistical variance, and results in a better recognition accuracy. However, due to the processing time, a tradeoff has to be considered between the time consumption and the classification accuracy. No explicit preference in the channel selection is possibly attributed to the preconditions of keeping all features extracted from all channels with $250 \mathrm{~ms}$, where the baseline of an acceptable recognition accuracy can be achieved.
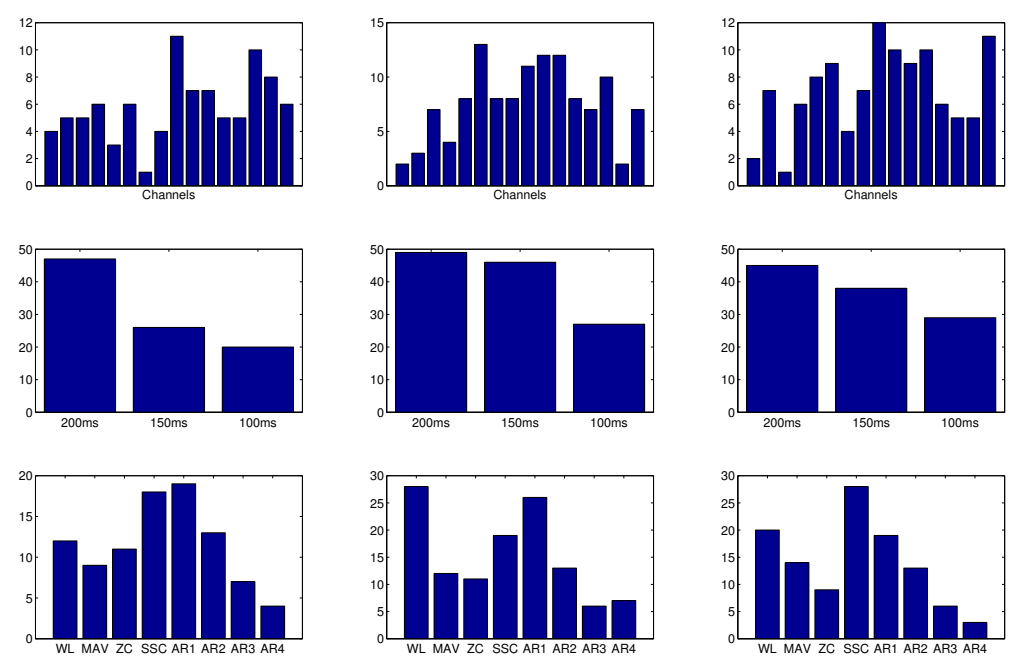

Fig. 7. Enumeration of the selected channels, window lengths, feature types

\section{Conclusion}

In this paper, the feature selection among segmentation sub-windows with multiple lengths was investigated in an inter-day hand motion recognition scenario. Improvement in the average recognition accuracy has been seen on the two subjects as a case study. The potential of multi-length windowed feature fusion was preliminarily shown. The enumeration of multiple feature selection process showed support to the longer window lengths, and feature types of waveform length, slope sign changes and the first 2 components of autoregressive coefficients. Our next work is to further apply the feature selection results in scenarios 
Table 1. Enumeration of window lengths and feature types

\begin{tabular}{|c|c|c|c|}
\hline Category & Candidate 1 & Candidate 2 & Candidate 3 \\
\hline Window length 200ms & 47 & 49 & 45 \\
\hline Window length 150ms & 26 & 46 & 38 \\
\hline Window length 100ms & 20 & 27 & 29 \\
\hline Feature WL & 12 & 28 & 20 \\
\hline Feature MAV & 9 & 12 & 14 \\
\hline Feature ZC & 11 & 11 & 9 \\
\hline Feature SSC & 18 & 19 & 28 \\
\hline Feature AR1 & 19 & 26 & 19 \\
\hline Feature AR2 & 13 & 13 & 13 \\
\hline Feature AR3 & 7 & 6 & 6 \\
\hline Feature AR4 & 4 & 7 & 3 \\
\hline
\end{tabular}

with variant arm positions, exerted forces and various levels of muscle fatigue. A supplementary dataset captured from amputee subjects will be considered to validate its clinical usability. Besides, the inter-subject recognition accuracy will be checked upon the incorporation of more subjects.

Acknowledgments. The authors would like to acknowledge support from DREAM project of EU FP7-ICT (grant no. 611391), and National Natural Science Foundation of China (grant no. 51575338 and 51575412).

\section{References}

1. Ji, X., Wang, C., Ju, Z.: A new framework of human interaction recognition based on multiple stage probability fusion. Applied Sciences 7(6) (2017) 567

2. Fang, Y., Hettiarachchi, N., Zhou, D., Liu, H.: Multi-modal sensing techniques for interfacing hand prostheses: a review. IEEE Sensors Journal 15(11) (2015) 6065-6076

3. Xue, Y., Ju, Z., Xiang, K., Chen, J., Liu, H.: Multimodal human hand motion sensing and analysis-a review. IEEE Transactions on Cognitive and Developmental Systems (2018)

4. Atzori, M., Cognolato, M., Müller, H.: Deep learning with convolutional neural networks applied to electromyography data: a resource for the classification of movements for prosthetic hands. Frontiers in neurorobotics 10 (2016) 9

5. Zhai, X., Jelfs, B., Chan, R.H., Tin, C.: Self-recalibrating surface emg pattern recognition for neuroprosthesis control based on convolutional neural network. Frontiers in neuroscience 11 (2017) 379

6. Erik Scheme MSc, P., Kevin Englehart PhD, P.: Electromyogram pattern recognition for control of powered upper-limb prostheses: State of the art and challenges for clinical use. Journal of Rehabilitation Research and Development 48(6) (2011) 643

7. Phinyomark, A., Quaine, F., Charbonnier, S., Serviere, C., Tarpin-Bernard, F., Laurillau, Y.: Emg feature evaluation for improving myoelectric pattern recognition robustness. Expert Systems with Applications 40(12) (2013) 4832-4840 
8. Oskoei, M.A., Hu, H.: Ga-based feature subset selection for myoelectric classification. In: 2006 IEEE International Conference on Robotics and Biomimetics, IEEE (2006) 1465-1470

9. Khushaba, R.N., Al-Jumaily, A.: Channel and feature selection in multifunction myoelectric control. In: Engineering in Medicine and Biology Society, 2007. EMBS 2007. 29th Annual International Conference of the IEEE, IEEE (2007) 5182-5185

10. Al-Timemy, A.H., Bugmann, G., Escudero, J., Outram, N.: Classification of finger movements for the dexterous hand prosthesis control with surface electromyography. IEEE Journal of Biomedical and Health Informatics 17(3) (2013) 608-618

11. Al-Angari, H.M., Kanitz, G., Tarantino, S., Cipriani, C.: Distance and mutual information methods for emg feature and channel subset selection for classification of hand movements. Biomedical Signal Processing and Control 27 (2016) 24-31

12. Adewuyi, A.A., Hargrove, L.J., Kuiken, T.A.: Evaluating emg feature and classifier selection for application to partial-hand prosthesis control. Frontiers in neurorobotics $10(2016) \quad 15$

13. Smith, L.H., Hargrove, L.J., Lock, B.A., Kuiken, T.A.: Determining the optimal window length for pattern recognition-based myoelectric control: balancing the competing effects of classification error and controller delay. IEEE Transactions on Neural Systems and Rehabilitation Engineering 19(2) (2011) 186-192

14. Zhou, D., Fang, Y., Botzheim, J., Kubota, N., Liu, H.: Bacterial memetic algorithm based feature selection for surface emg based hand motion recognition in long-term use. In: Computational Intelligence (SSCI), 2016 IEEE Symposium Series on, IEEE (2016) $1-7$

15. Hudgins, B., Parker, P., Scott, R.N.: A new strategy for multifunction myoelectric control. IEEE Transactions on Biomedical Engineering 40(1) (1993) 82-94

16. Botzheim, J., Cabrita, C., Kóczy, L.T., Ruano, A.: Fuzzy rule extraction by bacterial memetic algorithms. International Journal of Intelligent Systems 24(3) (2009) 312-339

17. Fang, Y., Liu, H., Li, G., Zhu, X.: A multichannel surface emg system for hand motion recognition. International Journal of Humanoid Robotics 12(02) (2015) 1550011

18. Englehart, K., Hudgins, B.: A robust, real-time control scheme for multifunction myoelectric control. IEEE Transactions on Biomedical Engineering 50(7) (2003) $848-854$ 